\title{
Captopril inhibits calpain-mediated apoptosis of myocardial cells in diabetic rats and improves cardiac function
}

\author{
LI-YA DONG $^{1 *}$, LI-PING YAO ${ }^{1 *}$, JING ZHAO $^{2}$, KE-KE JIN $^{3}$ and XIAO-XIAO QIU ${ }^{3}$ \\ ${ }^{1}$ Department of Cardiothoracic Surgery, Xinhua Hospital, School of Medicine, Shanghai Jiaotong University, \\ Shanghai 200092; ${ }^{2}$ Department of Pathophysiology, Anqing Medical College, Anqing, Anhui 246052; \\ ${ }^{3}$ Department of Pathophysiology, Wenzhou Medical University, Wenzhou, Zhejiang 325000, P.R. China
}

Received October 14, 2017; Accepted March 12, 2018

DOI: $10.3892 / \mathrm{mmr} .2018 .9192$

\begin{abstract}
To explore the effects of captopril on calpain-mediated apoptosis of myocardial cells and cardiac function in diabetic rats, 30 adult male Sprague-Dawley rats were randomly divided into three groups: Negative control (NC group), untreated diabetic rats (DM group) and diabetic rats treated with captopril (Cap group). Diabetes was induced by streptozotocin injection. Captopril was intragastrically administered at a daily dose of $50 \mathrm{mg} / \mathrm{kg}$ for 12 weeks; the $\mathrm{NC}$ and DM groups received an equivalent volume of saline. After 12 weeks of treatment, left ventricular systolic pressure (LVSP), left ventricular end-diastolic pressure (LVDEP), maximal rate of left ventricular pressure increase ( $+\mathrm{dp} / \mathrm{dtmax})$, maximal rate of left ventricular pressure decrease (-dp/dtmax) and left ventricular mass index (LVMI) were measured. The levels of calpain-1, calpain-2, B-cell lymphoma (Bcl)-2, Bcl-2 associated protein $\mathrm{X}$ (Bax) and total caspase-3 were detected in cardiac tissue by western blot analysis. The apoptotic index (AI) was assessed with a terminal deoxynucleotidyl transferase-mediated dUTP nick-end labeling assay. The ultrastructure of cardiac tissue was determined by transmission electron microscopy. Compared with the NC group,
\end{abstract}

Correspondence to: Dr Xiao-Xiao Qiu, Department of Pathophysiology, Wenzhou Medical University, University Town, Chashan, Wenzhou, Zhejiang 325000, P.R. China

E-mail: happy_qxx@163.com

${ }^{*}$ Contributed equally

Abbreviations: ACEI, angiotensin converting enzyme inhibitor; DM, diabetes mellitus; STZ, streptozotocin; LVSP, left ventricular systolic pressure; LVDEP, left ventricular end-diastolic pressure; $+\mathrm{dp} / \mathrm{dtmax}$, maximal rate of left ventricular pressure increase; -dp/dtmax, maximal rate of left ventricular pressure decrease; LVMI, left ventricular mass index; AI, apoptosis index; TUNEL, terminal deoxynucleotidyltransferase-mediated dUTP nick-end labeling; DC, diabetic cardiomyopathy; RAS, renin angiotensin system; Ang II, angiotensin II

Key words: calpain, diabetic cardiomyopathy, captopril, apoptosis
LVDEP and LVMI were increased, whereas LVSP, $+\mathrm{dp} / \mathrm{dtmax}$ and -dp/dtmax were decreased in the DM group. In the Cap group, LVDEP and LVMI were decreased, whereas LVSP, $+\mathrm{dp} / \mathrm{dtmax}$ and $-\mathrm{dp} / \mathrm{dtmax}$ were increased compared with the DM group. Bcl-2 protein expression was decreased, whereas the levels of calpain-1, calpain-2, Bax and total caspase-3 protein were increased in the DM group, compared with the $\mathrm{NC}$ group. Cap treatment increased $\mathrm{Bcl}-2$ protein expression and decreased calpain-1, calpain-2, Bax and total caspase-3 protein expression compared with the DM group. Additionally, the AI was increased in the DM group compared with the NC group, and decreased in the Cap group compared with the DM group. Furthermore, ultrastructural examination demonstrated that myocardial cell injury was reduced in the Cap group compared with the DM group. Therefore, captopril improved myocardial structure and ventricular function, by inhibiting calpain-1 and calpain-2 activation, increasing Bcl-2 expression, reducing Bax expression and subsequently inhibiting caspase-3-dependent apoptosis.

\section{Introduction}

Diabetic cardiomyopathy (DC) results in the development of cardiac microvascular lesions and myocardial structural disruption caused by metabolic disorder (1). DC is one of the most common diabetic complications and is pathologically characterized by the apoptosis and hypertrophy of myocardial cells, myocardial interstitial fibrosis and inflammation. DC may lead to heart failure, a major cause of death among patients with DM (2,3). However, the pathogenic mechanisms underlying the development of DC have not been fully elucidated.

Previous studies reported that cell apoptosis is involved in the occurrence and development of DC (4-6). Myocyte apoptosis caused by the long-term effects of high blood glucose comprises a cascade amplification reaction of caspase hydrolysate that is regulated as the genetic level; the B-cell lymphoma $(\mathrm{Bcl})$ family and caspase-3 serve important roles in apoptosis (4). Calpain, a member of the caspase superfamily, is a calcium-activated neutral protease. The earliest known members of the calpain family, calpain-1 and calpain-2, are the most extensively studied (7). Under certain pathological conditions, particularly a high-calcium environment, calpains are critical factors in inducing cell hypertrophy and/or death (8). 
Additionally, previous studies have revealed that upregulation of calpains in myocardial cells can lead to cell apoptosis $(9,10)$.

Angiotensin-converting enzyme inhibitors (ACEIs) can inhibit myocardial cell apoptosis in diabetic rats, significantly improve cardiac function and effectively reverse ventricular remodeling in DC $(11,12)$. However, whether ACEI regulates calpain-mediated apoptosis of myocardial cells and affects cardiac function in patients with DM remains unknown. It has been reported that angiotensin 2 receptor density increases in type 2 diabetic patients, and activation of the renin-angiotensin-aldosterone system can improve left ventricular function in patients with type $2 \mathrm{DM}$ (13). In the present study, the ACEI captopril was investigated in streptozotocin (STZ)-induced diabetic rats for its effects on the apoptosis of myocardial cells in DM, the expression of apoptotic proteins and left ventricular function.

\section{Materials and methods}

Ethical approval of the study protocol. The present study was approved by the Ethics Committee of Wenzhou Medical University (Wenzhou, China). All experimental procedures conformed to the guidelines for the Animal Care and Use Committee of Wenzhou Medical University (Wenzhou, China).

Reagents and instruments. Captopril was purchased as $25 \mathrm{mg}$ tablets from Changzhou Pharmaceutical Factory Co., Ltd. (Changzhou, China). STZ and sodium citrate were obtained from Sigma-Aldrich (Merck KGaA, Darmstadt, Germany). The terminal deoxynucleotidyltransferase-mediated dUTP nick end labeling (TUNEL) assay kit was purchased from Wuhan Boster Biological Technology, Ltd. (Wuhan, China). Antibodies against calpain-1 and calpain-2 and GAPDH were purchased from Genetimes Technology, Inc. (Shanghai, China); rabbit anti-mouse Bcl-2, Bcl-2 associated protein $\mathrm{X}$ (Bax), caspase-3 polyclonal antibodies and horseradish peroxidase (HRP)-tagged goat anti-rabbit secondary antibodies were obtained from OriGene Technologies, Inc. (Beijing, China). A bicinchoninic acid (BCA) protein assay kit was obtained from Pierce (Thermo Fisher Scientific, Inc., Waltham, MA, USA). Polyvinylidene fluoride (PVDF) membranes were purchased from Bio-Rad Laboratories, Inc. (Hercules, CA, USA) and a BeyoECL Plus Western Blotting chemiluminescence kit was purchased from Beyotime Institute of Biotechnology (Shanghai, China). The Johnson Sure Step ${ }^{\circledR}$ blood glucose meter and test strips were purchased from Johnson \& Johnson (New Brunswick, NJ, USA). The GB303 automatic electronic balance was obtained from Inesa Instrument Co., Ltd. (Shanghai, China) and the DH-140B animal ventilator was obtained from the Experimental Instrument Factory of Zhejiang Medical University (Hangzhou, China). The BL-420E data acquisition and processing system for bio-functional experiments was purchased from Chengdu Techman Software Co., Ltd. (Chengdu, China); the MUVB-20 gel imaging system was purchased from Ultra-Lum, Inc. (Claremont, CA, USA); the H-600 transmission electron microscope (TEM) was obtained from Hitachi, Ltd. (Tokyo, Japan); and the light microscope was obtained from Olympus Corporation (Tokyo, Japan).
Animal model and experimental protocol. A total of 30 healthy male specific pathogen-free (SPF) Sprague-Dawley rats (180-220 g; age, 2 months) were obtained from the Experimental Animal Center of Wenzhou Medical University (Wenzhou, China) and housed in an air-conditioned room at $23 \pm 2^{\circ} \mathrm{C}$ under a 12 -h light/dark cycle. Rats were randomly assigned to the normal control group $(\mathrm{NC} ; \mathrm{n}=10)$ and the diabetes group $(n=20)$. All animals were kept in an SPF environment and had access to food and water ad libitum. After a $12 \mathrm{~h}$ fast, rats of the diabetes group were intraperitoneally administered STZ $(65 \mathrm{mg} / \mathrm{kg})$ and the $\mathrm{NC}$ group rats received the equivalent volume of saline. At $72 \mathrm{~h}$ after the STZ injection, blood glucose was measured. Rats $(n=20)$ with a blood glucose level $>13.8 \mathrm{mmol} / \mathrm{l}$ were selected and assigned to the diabetes mellitus group (DM; $n=10)$ and the other half of the diabetic rats were treated with captopril (Cap; $n=10)$. Animals in the Cap group were intragastrically administered captopril daily $(50 \mathrm{mg} / \mathrm{kg}$ ) for 12 weeks; DM and NC groups were given normal saline at an equivalent volume. Additionally, the mental state, behavior, coat color/luster, consumption of feed and water, paving wetness degree by the urine were observed to analyze polydipsia, polyphagia and polyuria; body weight of the rats was monitored daily for 12 weeks. Postprandial blood glucose was measured every second week using a Johnson Sure Step ${ }^{\circledR}$ blood glucose meter, with blood sampled from the tail vein. At the end of week 12, left ventricular function was assessed using the BL-420E bio-functional experiment system. Following this, all animals were sacrificed, hearts were harvested and stored in an ice bath. The hearts and left ventricles were weighed. Subsequently, the left ventricular tissues were used for making paraffin sections (4-6 $\mu \mathrm{m}$ ) and electron microscope specimens or stored immediately in $-70^{\circ} \mathrm{C}$ for western blotting.

Left ventricular function and left ventricular mass index (LVMI). The animals were intraperitoneally anesthetized with $10 \%$ chloral hydrate $(350 \mathrm{mg} / \mathrm{kg})$. A tracheal cannula was inserted into the rats and connected to a DH-140B animal ventilator; the tidal volume was $5 \mathrm{ml}$ and the respiratory rate was 55 breaths per minute, with an inspiration/expiration ratio of $1.5: 1$. A cardiac catheter was subsequently inserted into the left ventricle to determine the left ventricular systolic pressure (LVSP), left ventricular end-diastolic pressure (LVDEP), maximal rate of left ventricular pressure increase (+dp/dtmax) and maximal rate of left ventricular pressure decrease (-dp/dtmax). Following this, rats were sacrificed to harvest the heart and the left ventricle was isolated. The mass of the left ventricle was measured. The LVMI was calculated as the mass of the left ventricle/mass of the heart.

Western blot analysis. The expression of calpain-1, calpain-2, Bcl-2, Bax and caspase-3 was examined by western blotting. Briefly, total protein of the heart tissue was extracted with ice-cold radioimmunoprecipitation assay lysis buffer supplemented with PMSF for $30 \mathrm{~min}$, the whole-cell lysate and the concentration of total protein was determined by the BCA method (14). Equal amounts of protein $(20 \mu \mathrm{g})$ were separated by SDS-PAGE (5\% stacking gel and $12 \%$ separating gel) and transferred to PVDF membranes. The membranes were 


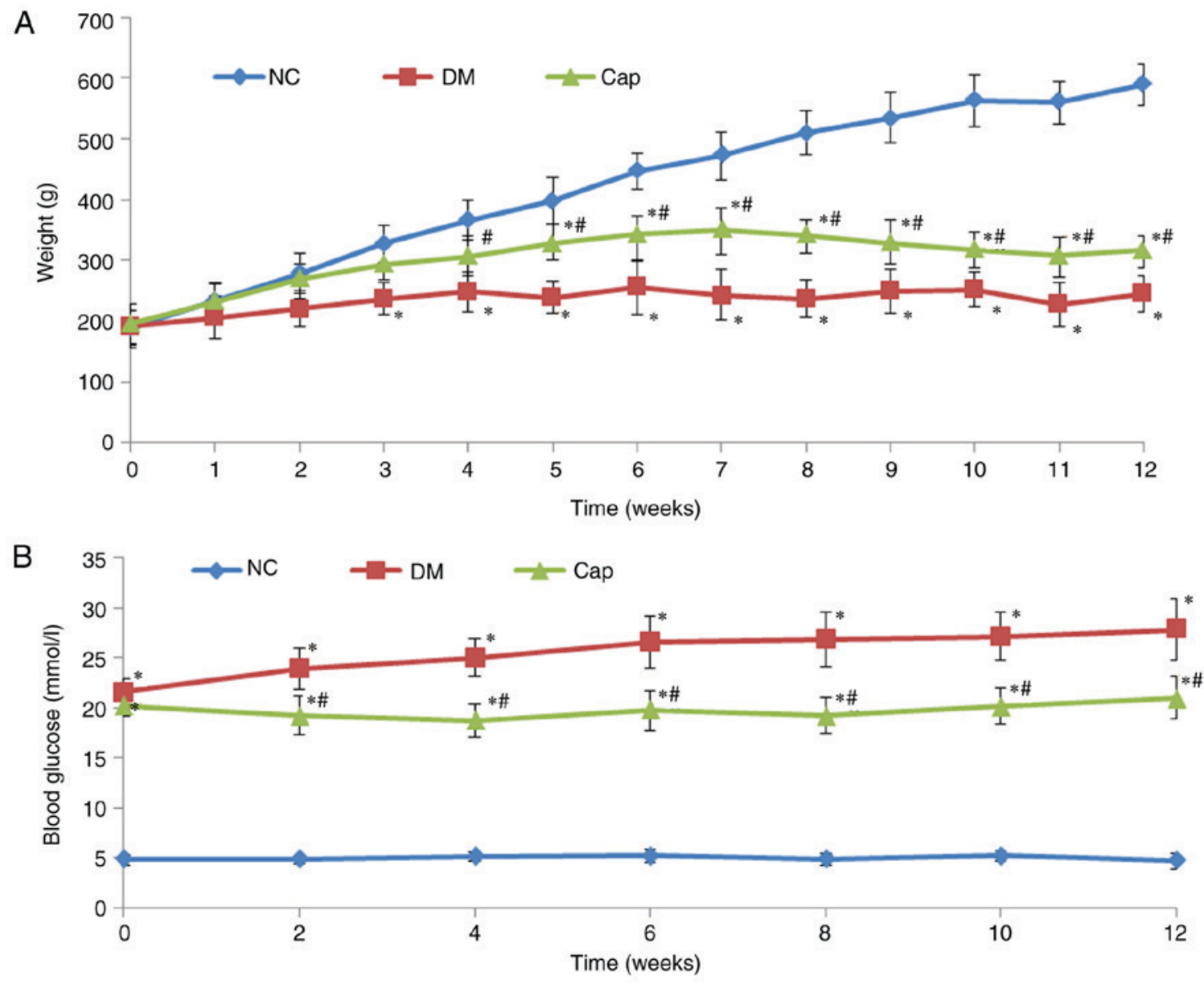

Figure 1. Alterations in (A) body weight and (B) glucose levels in diabetic rats treated with or without Cap for 12 weeks. NC group rats received an equivalent volume of saline. Each group, $\mathrm{n}=10$ rats. Values are presented as the mean \pm standard deviation. ${ }^{*} \mathrm{P}<0.05$ vs. NC group, ${ }^{\#} \mathrm{P}<0.05$ vs. DM group. Cap, captopril; NC, negative control; DM, diabetes mellitus.

blocked in $5 \%$ non-fat dry milk for $1 \mathrm{~h}$ at room temperature and subsequently incubated overnight at $4^{\circ} \mathrm{C}$ with calpain-1 large-subunit antibody (1:400), calpain-2 large-subunit antibody (1:400), rabbit anti-mouse Bcl-2 (1:250), Bax polyclonal antibody (1:400) and caspase-3 polyclonal antibody (1:600). The membrane was rinsed in Tris-buffered saline with $0.05 \%$ Tween 20 prior to incubation with HRP-labeled secondary antibody (1:5,000; cat. no. 111-035-006) at room temperature for $2 \mathrm{~h}$. Protein bands were visualized with the BeyoECL Plus Western Blotting chemiluminescence kit and analyzed with Quantity One software (version 4.52, Bio-Rad Laboratories, Inc.). GAPDH (1:1,000; cat. no. 5174) was included in as an internal reference for the quantification of relative protein expression.

Myocardial apoptosis and apoptotic index (AI). Apoptotic myocardial cells were detected by a TUNEL assay kit according to the manufacturer's instructions (Wuhan Boster Biological Technology, Ltd.). Paraffin sections of the heart tissues were dewaxed, rinsed in water and PBS, and treated with protease $\mathrm{K}$ solution at $37^{\circ} \mathrm{C}$ for $15 \mathrm{~min}$. Tagging buffer $(20 \mu \mathrm{l})$ was added at $37^{\circ} \mathrm{C}$. After $60 \mathrm{~min}$, samples were rinsed with PBS, stained with $0.05 \%$ 3'3'-Diaminobenzidine for $10 \mathrm{~min}$ at room temperature and counterstained with $0.025 \%$ hematoxylin for $1 \mathrm{~min}$ at room temperature. Positive cells (apoptotic) were characterized by brown granules inside the nucleus. Apoptotic cells in five random high-power visual fields (magnification, x400) were counted under a light microscope. AI (\%) was determined as the apoptotic cell count/100 cells (15).

Myocardial ultrastructure observation. Myocardial tissue $\left(1 \times 1 \times 1 \mathrm{~mm}^{3}\right)$ was collected from the anterior wall of the left ventricle, pre-fixed in $2.5 \%$ glutaraldehyde and post-fixed in $1 \%$ osmic acid at $37^{\circ} \mathrm{C}$ for $60 \mathrm{~min}$. Samples were subsequently dehydrated with a gradient of acetone $(50 \%$ acetone for $10 \mathrm{~min}, 70 \%$ acetone 10 for $\mathrm{min}, 80 \%$ acetone for $10 \mathrm{~min}, 90 \%$ acetone for $10 \mathrm{~min}$ and twice with $100 \%$ acetone for $10 \mathrm{~min}$ ), embedded in Epon $812\left(45^{\circ} \mathrm{C}\right.$ for $6 \mathrm{~h}$ then $65^{\circ} \mathrm{C}$ for $\left.48 \mathrm{~h}\right)$ and sliced into ultrathin sections $(1 \mu \mathrm{m})$. Samples were double stained with $4 \%$ lead nitrate for $10 \mathrm{~min}$ at room temperature and uranyl acetate $30 \mathrm{~min}$ at room temperature and observed by TEM.

Statistical analysis. SPSS 17.0 software (SPSS, Inc., Chicago, IL, USA) was used to perform the statistical analyses. All data are expressed as the mean \pm standard deviation. The data in each group were subjected to normality testing and homogeneity of variance was analyzed. Inter-group comparisons were performed by one-way analysis of variance. Pairwise comparisons between groups with homogeneous variance were performed with LSD method, whereas comparisons 
Table I. Comparison of cardiac function among different groups in rats.

\begin{tabular}{lccccc}
\hline Group & LVSP $(\mathrm{mmHg})$ & LVDEP $(\mathrm{mmHg})$ & + dp/dtmax $(\mathrm{mmHg} / \mathrm{s})$ & $-\mathrm{dp} / \mathrm{dtmax}(\mathrm{mmHg} / \mathrm{s})$ & $\mathrm{LVMI}(\mathrm{mg} / \mathrm{g})$ \\
\hline NC & $118.20 \pm 10.83$ & $3.57 \pm 1.19$ & $5,382.43 \pm 693.10$ & $5,310.53 \pm 696.23$ & $1.53 \pm 0.16$ \\
DM & $90.20 \pm 8.87^{\mathrm{a}}$ & $10.80 \pm 3.37^{\mathrm{a}}$ & $3,783.52 \pm 863.27^{\mathrm{a}}$ & $3,725.95 \pm 864.76^{\mathrm{a}}$ & $2.25 \pm 0.30^{\mathrm{a}}$ \\
Cap & $103.41 \pm 9.36^{\mathrm{b}}$ & $5.10 \pm 1.21^{\mathrm{b}}$ & $47,89.99 \pm 707.06^{\mathrm{b}}$ & $5,024.43 \pm 573.60^{\mathrm{b}}$ & $1.82 \pm 0.21^{\mathrm{b}}$ \\
\hline
\end{tabular}

${ }^{\mathrm{a}} \mathrm{P}<0.05$ vs. NC group, ${ }^{\mathrm{b}} \mathrm{P}<0.05$ vs. DM group. NC, negative control; DM, diabetes mellitus; Cap, captopril; LVSP, left ventricular systolic pressure; LVDEP, left ventricular end-diastolic pressure; +dp/dtmax, maximal rise rate of left ventricular pressure; -dp/dtmax, maximal fall rate of left ventricular pressure; LVMI, left ventricular mass index. Data are presented as the mean \pm standard deviation.

between groups with homogeneous variance were performed with Dunnett's T3 test. $\mathrm{P}<0.05$ was considered to indicate a statistically significant difference.

\section{Results}

Captopril treatment increases body weight and reduces glucose levels in diabetic rats. Compared with the DM group, animals in the Cap group exhibited significant improvement in DM-associated symptoms, including polydipsia, polyphagia and polyuria. Cap rats were relatively active and their coats regained luster. The body weights in the DM and Cap groups were significantly decreased compared with those in the NC group. Furthermore, the body weights in the Cap group were significantly increased compared with the DM group $(\mathrm{P}<0.05$; Fig. 1A). Blood glucose at 12 weeks was significantly increased in the DM and Cap groups, compared with the NC group $(\mathrm{P}<0.05)$. Cap group glucose levels were significantly decreased compared with those in the DM group ( $\mathrm{P}<0.05$; Fig. 1B).

Captopril treatment improves cardiac function in diabetic rats. Compared with the NC group, DM rats had significantly increased LVDEP and LVMI. LVSP, +dp/dtmax and -dp/dtmax were significantly decreased in the DM group, compared with the NC group. Furthermore, compared with the DM group, Cap-treated rats had significantly lower LVDEP and LVMI. LVSP, $+\mathrm{dp} / \mathrm{dtmax}$ and -dp/dtmax significantly increased compared with the DM group (Table I).

Captopril reduces cell apoptosis in myocardial tissue. In the NC group, cell apoptosis was rarely observed. Compared with the NC group, apoptosis in the DM group increased by $\sim 15$-fold $(\mathrm{P}<0.05)$. Captopril treatment decreased the number of apoptotic cells by $24 \%$, compared with the DM group (P<0.05; Fig. 2).

Calpain-1, calpain-2, Bcl-2, Bax and total caspase-3 expression alterations in heart tissue in response to captopril. Compared with the NC group, Bcl-2 expression was significantly decreased in the DM group, while calpain-1, calpain-2, Bcl-2, Bax and total caspase-3 expression significantly increased $(\mathrm{P}<0.05)$. By contrast, captopril treatment significantly increased $\mathrm{Bcl}-2$ expression and significantly reduced calpain-1, calpain-2, Bax and total caspase-3 expression compared with the DM group ( $\mathrm{P}<0.05$; Fig. 3$)$.
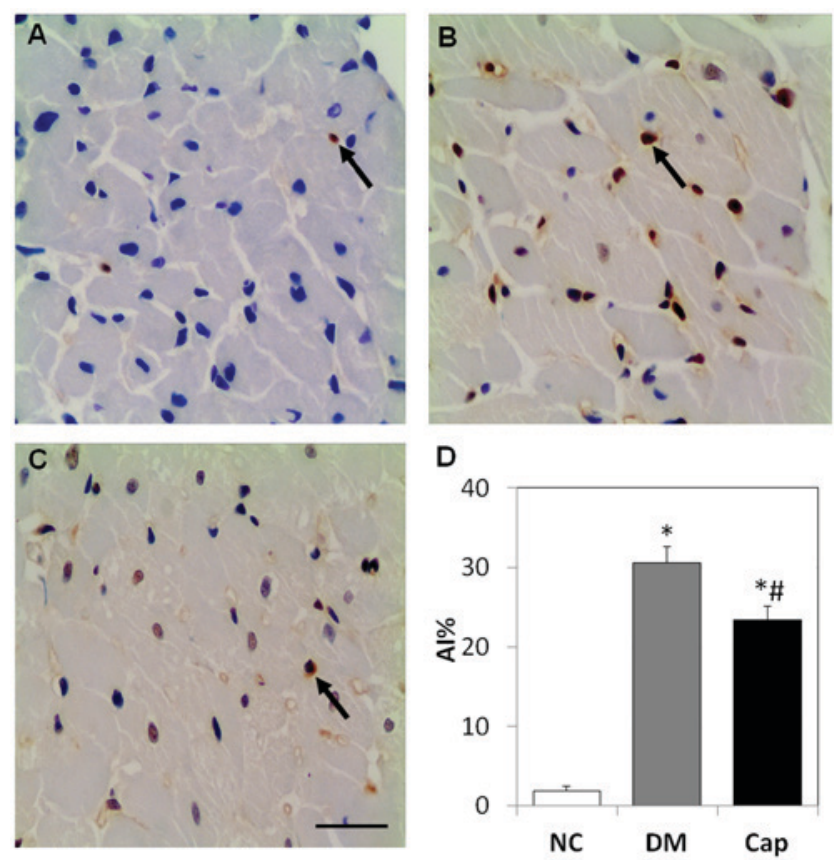

Figure 2. Cell apoptosis in cardiac tissue. Arrows indicate representative apoptotic cells. Myocardial apoptosis in the (A) NC, (B) DM and (C) Cap groups. (D) The AI was calculated. Values are presented as the mean \pm standard deviation. Each group, $n=10$. Magnification, $x 400$; scale bar, $50 \mu \mathrm{m} .{ }^{*} \mathrm{P}<0.05$ vs. NC group, ${ }^{*} \mathrm{P}<0.05$ vs. DM group. $\mathrm{NC}$, negative control; DM, diabetes mellitus; Cap, captopril; AI, apoptotic index.

Alterations in myocardial ultrastructure in response to captopril. The myocardium of rats in the NC group exhibited solid myofibrils and regularly distributed myofilaments. The mitochondrial membranes were intact and the arrangement of the ridges was regular. The basilar membranes of the microvessels were continuous and intact (Fig. 4A).

In the myocardium of the DM group, the myocytes were swollen and punctiform myofibril dissolution was observed. The arrangement of the myofilaments was irregular and myofilaments were partially disrupted. The mitochondria were enlarged and the ridge arrangement was disrupted, with the presence of vacuoles. Interstitial collagen hyperplasia was observed and the basement membranes of the microvessels were thickened (Fig. 4B).

In the Cap group, the myofibrils were partially disrupted. Mitochondrial enlargement was alleviated. Minor disruptions were observed in the ridges and the basement membrane thickening of the microvessels was attenuated (Fig. 4C). 
A

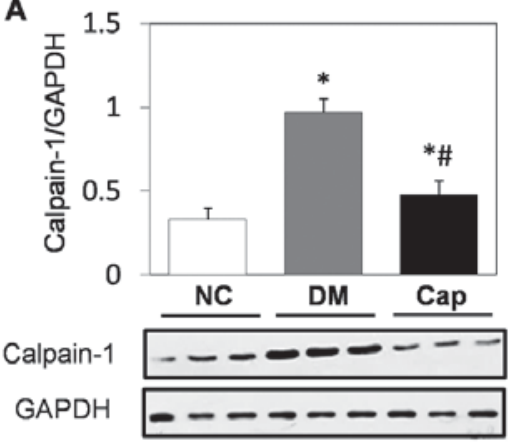

B

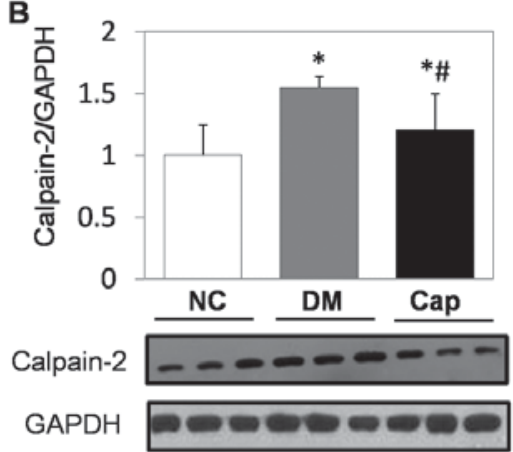

$$
\text { C }
$$

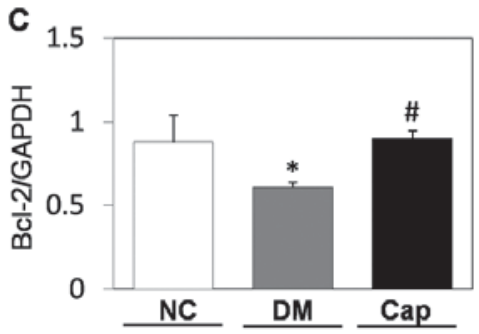

$\mathrm{Bcl}-2$

GAPDH

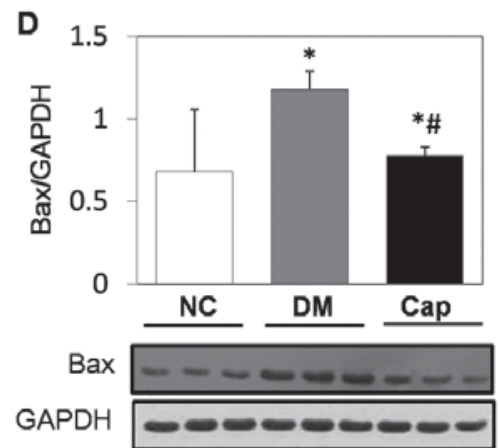

Figure 3. Western blot analysis of apoptotic protein expression. Protein expression of (A) calpain-1, (B) calpain-2, (C) Bcl-2, (D) Bax and (E) caspase-3 in cardiomyocytes from NC, DM and Cap-treated rats. GAPDH was used as the internal reference protein. Data are expressed as the mean \pm standard deviation. $\mathrm{P}<0.05$ vs. NC group, ${ }^{\#} \mathrm{P}<0.05$ vs. DM group. Bcl-2, B-cell lymphoma 2; Bax, Bcl-2 associated protein X; NC, negative control; DM, diabetes mellitus; Cap, captopril.
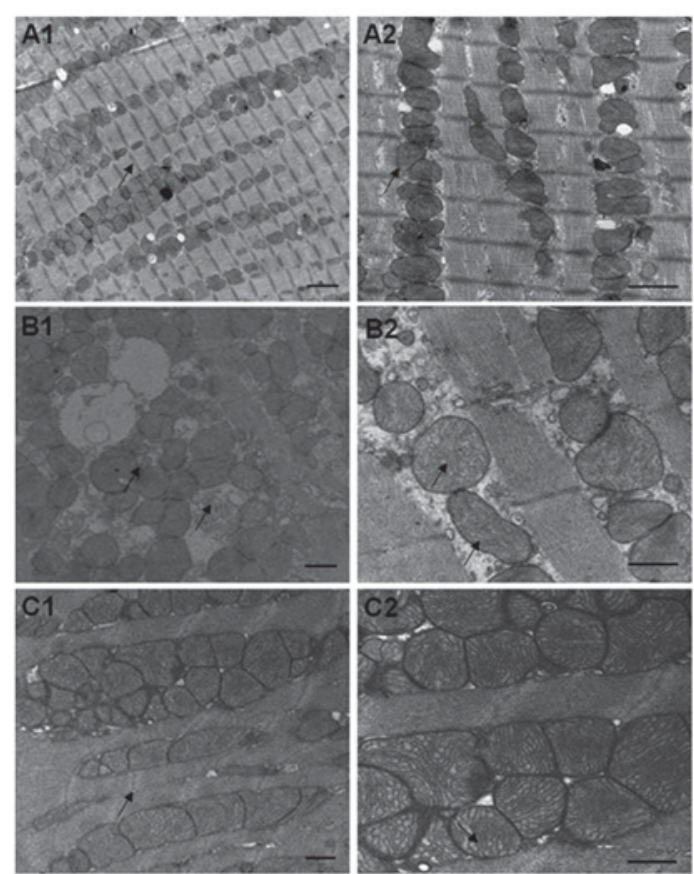

Figure 4. Representative images of transmission electron microscopic observation of cardiomyocyte ultrastructure. (A) Myocardium of rats in the NC, (B) DM and (C) Cap groups. Scale bar, $1 \mu \mathrm{m}$. Magnification A1, B1 and $\mathrm{C} 1 \times 5,000 ; \mathrm{A} 2, \mathrm{~B} 2$ and $\mathrm{C} 2 \times 10,000$. (A1) The arrangement of myofilaments was regular and dense; (A2) The Mitochondria were closely arranged and uniform in density. (B1) The arrangement of the myofilaments was irregular and myofilaments were partially disrupted. The mitochondria were enlarged and the arrangement was disorderly; (B2) The mitochondria ridge was arranged disorderly and even disrupted. The myofibrillar gap became widening. (C1) The arrangement of the myofilaments was partially irregular, The myofibrillar gap was not obvious widening; (C2) The mitochondria were mildly enlarged, the ridge was rich and regular.

\section{Discussion}

In the present study, it was demonstrated that treatment with captopril increased body weight and reduced blood glucose in diabetic rats. This result was consistent with a previous study reporting that captopril can protect islet function, prevent diabetes occurrence and improve diabetic rat weight loss $(16,17)$. In the present study, captopril improved cardiac function, inhibited myocardial cell apoptosis and protected myocardial structure, thereby improving ventricular function. This was likely achieved by reducing the activation of calpain-1, calpain-2 and Bax, as well as upregulating the expression of Bcl-2, leading to the inhibition of caspase-3-dependent apoptosis.

At week 12, STZ-induced diabetic rats in the current study exhibited abnormalities in the systole and diastole of the left ventricle and structural damage in the myocardium, including a significant increase in myocardial cell apoptosis. This was consistent with a previous study demonstrating that left ventricular systolic and diastolic dysfunction in DC is associated with myocardial cell apoptosis (18). The mechanism underlying the diabetes-induced apoptosis of myocytes is complex, involving the $\mathrm{Bcl}-2$ and caspase gene families $(19,20)$. Bcl-2 was the first anti-apoptotic gene discovered in humans (21). Although Bax is also a member of the Bcl-2 family, it functions as a pro-apoptotic gene. High Bcl-2 expression may lead to the formation of $\mathrm{Bcl}-2 / \mathrm{Bcl}-2$ homodimers and Bcl-2/Bax heterodimers, both of which have anti-apoptotic effects; however, high expression of Bax may yield Bax/Bax homodimers, which are pro-apoptotic (21). Whether apoptosis occurs in a certain group of cells and how much they are affected is determined 
by the ratio of Bcl-2 and Bax expression (22). In the present study, it was demonstrated that Bcl-2 was significantly decreased and Bax significantly increased in the DM group, compared with the NC group. This is in accordance with the results reported by Kumar et al (23), which revealed that the number of apoptotic myocardial cells increases in DM, along with a decrease in $\mathrm{Bcl}-2$ expression and increase in Bax expression.

The caspase family is a group of proteins critical for regulating and executing cell apoptosis. Caspase-3 is referred to as the 'death protease', as its activated form catalyzes the hydrolysis of specific proteins to promote apoptosis $(24,25)$. In the present study, apoptosis induced by hyperglycemia is regulated by the caspase-3-dependent mitochondrial pathway. Cai et al (26) demonstrated that high glucose-induced cell apoptosis occurs at least partially via a caspase-3-dependent mitochondrial pathway.

Calpains are a family of calcium-dependent cysteine proteases in the cytoplasm that are distributed widely in most mammals. Calpain-1 and calpain-2 are the most extensively studied members of this family; both are heterodimers composed of 28 and $80 \mathrm{kDa}$ subunits and may be expressed in myocytes. Their sequences share 55-65\% homology. Calpain-1 and -2 differ in that they require different calcium concentrations for their activation; $\mu$ mol levels for calpain- 1 and mmol levels for calpain-2. Typically, $\mathrm{Ca}^{2+}$ concentration in myocytes is not sufficient to activate these calpains (27). However, high glucose may increase the calcium load in myocytes, which is associated with a decrease in the activity of the $\mathrm{Na}^{+} / \mathrm{Ca}^{2+}$ exchanging ATPase and the $\mathrm{Ca}^{2+} \mathrm{ATPase}$, and subsequently inhibits the calcium concentration resurge in the sarcoplasmic reticulum (28). As revealed by a previous report (27), high glucose may lead to the production of reactive oxygen species, causing the activation of L-type calcium channels and ryanodine receptors and increase of in intracellular calcium concentrations, which subsequently leads to the activation of calpain. Activated calpain mediates the apoptosis of myocytes under high-glucose conditions via the caspase-3 pathway. An in vitro experiment demonstrated that pro-apoptotic Bax maybe cleaved by calpain to yield an $18 \mathrm{kDa}$ active fragment, which induces the release of mitochondrial cytochrome $c$ to mediate cell apoptosis (29). Therefore, the Bcl-2 family is involved in the pro-apoptotic effects of calpain, and several members of the Bcl-2 family are substrates of calpain (30). In the present research, compared with the control group, Bcl-2 expression was downregulated in myocardial tissue, whereas calpain-1, calpain-2, Bax and total caspase-3 expression was increased in the DM group, indicating that cell apoptosis in DC may be associated with the alterations in the expression of these proteins. This is consistent with previous reports (21-23). Furthermore, activation of calpain-1 and calpain-2 may mediate caspase-3-dependent apoptosis by downregulating $\mathrm{Bcl}-2$ and upregulating Bax to further induce the occurrence of DC.

ACEIs, including captopril, are considered protective agents against pancreatic dysfunction and diabetes (16). In the present study, captopril treatment alleviated the symptoms of DM rats. Local overactivation of the renin/angiotensin system (RAS) and dysfunction of angiotensin II (Ang II) are thought to be involved in DC apoptosis (31). Captopril may inhibit cell apoptosis by blocking local or systemic activation of RAS and inhibiting the bio-synthesis of Ang II (32). In the present study, increased expression of Bcl-2 and decreased expression of calpain-1, calpain-2, Bax and caspase-3 were observed in the myocardial tissue. Additionally, cell apoptosis was significantly inhibited, LVMI was decreased and systolic function was significantly improved. Furthermore, the ultrastructural damage in the myocardium was alleviated, suggesting that captopril may improve ventricular function and protect the myocardium by inhibiting the activation of calpain-1 and calpain-2, upregulating Bcl-2 and downregulating Bax to inhibit caspase-3-dependent myocyte apoptosis. A previous study reported similar functions of ACEI and calcium antagonists (33), both of which can ameliorate calcium overload. Therefore, ACEIs may inhibit calpain activation through the attenuation of calcium overload. However, further confirmation of this hypothesis is required.

In conclusion, the present study demonstrated that captopril may preserve myocardial function in diabetic rats via inhibition of cardiac cell apoptosis, suggesting that ACEIs, including captopril, maybe considered as a therapeutic option in DC.

\section{Acknowledgements}

We thank Professor Jianmin $\mathrm{Li}$, a pathological specialist from Wenzhou Medical University (Wenzhou, China) for technical assistance in this study. We are deeply grateful to Fanyan Wang and Lei Ying, Pathophysiological doctors from Wenzhou Medical University for their kind assistance in proofreading the manuscript.

\section{Funding}

This investigation was supported by a grant from National Nature Science Foundation of China (grant no. 81170204) and College Students' Science and Technology Innovation Foundation of Zhejiang Province (grant no. 2010R413002).

\section{Availability of data and materials}

The datasets used and analyzed during the current study are available from the corresponding author on reasonable request.

\section{Author's contributions}

LYD, LPY and XXQ designed the study, drafted the manuscript and approved its final version. JZ acquired data and revised the article for important intellectual content. KKJ was involved in generating the idea and gave final approval of the version to be published. All authors read and approved the final manuscript.

\section{Ethics approval and consent to participate}

The present study was approved by the Ethics Committee of Wenzhou Medical University. All experimental procedures conformed to the guidelines for the Animal Care and Use Committee of Wenzhou Medical University. 


\section{Consent for publication}

Not applicable.

\section{Competing interests}

The authors declare that they have no competing interests.

\section{References}

1. Mazzone T, Chait A and Plutzky J: Cardiovascular disease risk in type 2 diabetes mellitus: Insights from mechanistic studies. Lancet 371: 1800-1809, 2008.

2. Miettinen H, Lehto S, Salomma V, Mähönen M, Niemelä M, Haffner SM, Pyörälä K and Tuomilehto J; The FINMONICA Myocardial Infarction Register Study Group: Impact of diabetes on mortality after the first myocardial infarction. Diabetes Care 21: 69-75, 1998.

3. De Groote P, Lambin N, Mouquet F, Plichon D, McFadden E, Van Belle E and Bauters C: Impact of diabetes mellitus on long-term survival in patients with congestive heart failure. Eur Heart J 25: 656-662, 2004

4. Cai L and Kang YJ: Cell death and diabetic cardiomyopathy. Cardiovasc Toxicol 3: 219-228, 2003.

5. Li Z, Zhang T, Dai H, Liu G, Wang H, Sun Y, Zhang Y and Ge Z: Endoplasmic reticulum stress is involved in myocardial apoptosis of streptozocin-induced diabetic rats. J Endocrinol 196: 565-572, 2008.

6. Ou HC, Tzang BS, Chang MH, Liu CT, Liu HW, Lii CK, Bau DT, Chao PM and Kuo WW: Cardiac contractile dysfunction and apoptosis in streptozotocin-induced diabetic rats are ameliorated by garlic oil supplementation. J Agric Food Chem 58: 10347-10355, 2010.

7. Goll DE, Thompson VF, Li H, Wei W and Cong J: The calpain system. Physiol Rev 83: 731-801, 2003.

8. Inserte J, Garcia-Dorado D, Hernando V and Soler-Soler J: Calpain-mediated impairment of $\mathrm{Na}+\mathrm{K}+-\mathrm{ATPase}$ activity during early reperfusion contributes to cell death after myocardial ischemia. Circ Res 97: 465-473, 2005.

9. Galvez AS, Diwan A, Odley AM, Hahn HS, Osinska H, Melendez JG, Robbins J, Lynch RA, Marreez Y and Dorn GW II: Cardiomyocyte degeneration with calpain deficiency reveals a critical role in protein homeostasis. Circ Res 100: 1071-1078, 2007.

10. Bajaj G and Sharma RK: TNF-alpha-mediated cardiomyocyte apoptosis involves caspase-12 and calpain. Biochem Biophys Res Commun 345: 1558-1564, 2006.

11. Zhang CH, Lu J, Yu XJ, Sun L and Zang WJ: Ameliorative effect of Captopril and Valsartan on an animal model of diabetic cardiomyopathy. Biol Pharm Bull 31: 2045-2049, 2008.

12. Guan SJ, Ma ZH, Wu YL, Zhang JP, Liang F, Weiss JW, Guo QY, Wang JY, Ji ES and Chu L: Long-term administration of fasudil improves cardiomyopathy in streptozotocin-induced diabetic rats. Food ChemToxicol 50: 1874-1882, 2012

13. Symeonides P, Koulouris S, Vratsista E, Triantafyllou K, Ioannidis $\mathrm{G}$, Thalassinos $\mathrm{N}$ and Katritsis $\mathrm{D}$ : Both ramipril and telmisartan reverse indices of early diabetic cardiomyopathy. A comparative study. Eur J Echocardiogr 8: 480-486, 2007.

14. Akins RE and Tuan RS: Measurement of protein in 20 seconds using a microwave BCA assay. Biotechniques 12: 496-499, 1992.
15. Gavrieli Y, Sherman Y and Ben-Sasson SA: Identification of programmed cell death in situ via specific labeling of nuclear DNA fragmentation. J Cell Biol 119: 493-501, 1992.

16. Solski LV and Longyhore DS: Prevention of type 2 diabetes mellitus with angiotensin-converting-enzyme inhibitors. Am J Health Syst Pharm 65: 935-940, 2008.

17. Rodrigues de Araujo G, Granato de Faria K, Lima WG, Pádua Bda C, Rossoni JV Jr, Souza AA, Chianca-Júnior D, Silva ME, Pedrosa ML, Chaves MM and Costa DC: Effect of captopril and the bradykinin-PKC pathway on ROS production in type 1 diabetic rats. Can J Physiol Pharmacol 89: 923-933, 2011

18. Okoshi K, Guimarães JF, Di Muzio BP, Fernandes AA and Okoshi MP: Diabetic cardiomyopathy. Arq Bras Endocrinol Metabol 51: 160-167, 2007 (In Portuguese).

19. Bojunga J, Nowak D, Mitrou PS, Hoelzer D, Zeuzem S and Chow KU: Antioxidative treatment prevents activation of death-receptor- and mitochondrion-dependent apoptosis in the hearts of diabetic rats. Diabetologia 47: 2072-2080, 2004

20. Li CJ, Zhang QM, Li MZ, Zhang JY, Yu P and Yu DM: Attenuation of myocardial apoptosis by alpha-lipoic acid through suppression of mitochondrial oxidative stress to reduce diabetic cardiomyopathy. Chin Med J (Engl) 122: 2580-2586, 2009.

21. Cory S and Adams JM: The Bcl2 family: Regulators of the cellular life-or-death switch. Nat Rev Cancer 2: 647-656, 2002.

22. Walensky LD: Bcl-2 in the crosshairs: Tipping the balance of life and death. Cell Death Differ 13: 1339-1350, 2006.

23. Kumar S, Prasad S and Sitasawad SL: Multiple antioxidants improve cardiac complications and inhibit cardiac cell death in streptozotocin-induced diabetic rats. PLoS One 8: e67009, 2013.

24. Boatright KM and Salvesen GS: Caspase acrivation. Biochem Soc Symp: 233-242, 2003

25. Donovan $\mathrm{M}$ and Cotter TG: Control of mitochondrial integrity by Bcl-2 family members and caspase-independent cell death. Biochim Biophys Acta 1644: 133-147, 2004.

26. Cai L, Li W, Wang G, Guo L, Jiang Y and Kang YJ: Hyperglycemia-induced apoptosis in mouse myocardium: Mitochondrial cytochrome C-mediated caspase-3 activation pathway. Diabetes 51: 1938-1948, 2002.

27. Cesario DA, Brar R and Shivkumar K: Alterations in ion channel physiology in diabetic cardiomyopathy. Endocrinol Metab Clin North Am 35: 601-610, 2006.

28. Li Y, Li Y, Feng Q, Arnold M and Peng T: Calpainactivation contributes to hyperglycaemia-induced apoptosis in cardiomyocytes. Cardiovasc Res 84: 100-110, 2009.

29. Gao G and Dou QP: N-terminal cleavage of bax by calpain generates a potent proapoptotic $18-\mathrm{kDa}$ fragment that promotes bcl-2-independent cytochrome $\mathrm{C}$ release and apoptotic cell death J Cell Biochem 80: 53-72, 2000.

30. Gil-Parrado S, Fernández-Montalván A, Assfalg-Machleidt I, Popp O, Bestvater F, Holloschi A, Knoch TA, Auerswald EA, Welsh K, Reed JC, et al: Ionomycin-activated calpain triggers apoptosis. A probable role for Bcl-2 family members. J Biol Chem 277: 27217-27226, 2002.

31. Zhang CH, Lu J, Yu XJ, Sun L and Zang WJ: Ameliorative effect of Captopril and Valsartan on an animal model of diabetic cardiomyopathy. Biol Pharm Bull 31: 2045-2049, 2008.

32. Connelly KA, Boyle AJ and Kelly DJ: Angiotensin II and the cardiac complications of diabetes mellitus. Curr Pharm Des 13: 2721-2729, 2007

33. Zhu Z, Tepel M, Neusser M, Mehring N and Zidek W: Effect of captopril on vasoconstriction and $\mathrm{Ca} 2+$ fluxes in aortic smooth muscle. Hypertension 22: 806-811, 1993. 